\title{
Mode stabilized terrace InGaAsP lasers on semi-insulating InP
}

\author{
T. R. Chen, a) K. L. Yu, U. Koren, A. Hasson, S. Margalit, and A. Yariv \\ California Institute of Technology, Pasadena, California 91125
}

(Received 26 April 1982; accepted for publication 2 August 1982)

Mode stabilized terrace InGaAsP lasers have been fabricated on semi-insulating InP substrates.

The fabrication involves a selective, single-step liquid phase epitaxial growth process, and a lateral

$\mathrm{Zn}$ diffusion. Two versions of the terrace lasers are fabricated, and threshold currents as low as 35

$\mathrm{mA}$ and $50 \mathrm{~mA}$ respectively are obtained. The lasers operate with a stable single lateral mode.

High power performance is observed. These lasers are suitable for monolithic integration with

other optoelectronic devices.

PACS numbers: 42.55.Px, 42.60.By

Mode stabilization of semiconductor laser diode is of key importance in applications such as optical communication and signal processing. One of the basic approaches to laser mode stabilization is the creation of a built-in real index waveguiding structure. ${ }^{1}$ However, in strongly guiding dielectric waveguide laser such as buried heterostructure laser, ${ }^{2}$ or a groove laser, ${ }^{3}$ single transverse mode can be obtained only when the width of the active region is narrower than $\sim 2 \mu \mathrm{m}$. ${ }^{2}$ It is generally believed that weakly waveguiding structures are the preferred method for achieving single mode operation.

The terrace structure on conductive substrates has been reported for both the GaAs/GaAlAs and InGaAsP/InP systems. ${ }^{4,5}$ In that structure, due to the geometry of the terrace substrate, a part of the active layer is grown thicker than the rest to provide the built-in index waveguiding. The resultant lasers operate in stabilized single transverse mode. However, there is current leakage in the first cladding layer, either in the form of current spreading away from the stripe region, or in the form of parasitic junctions. Moreover, the lasers are grown on conductive substrates. Opto-electronic integration with other electronic devices is harder to achieve.

In this work we report on terrace structure lasers grown on semi-insulating (SI) InP. The present laser structure has the minimal current spreading while still maintaining the built-in weak index waveguiding characteristics, with stable single transverse mode behavior of the optical output maintained at over 10 times threshold current. As the lasers are fabricated on SI materials, opto-electronic integration with other devices is possible.

The laser structure reported here is grown using a selective, single-step liquid phase epitaxy (LPE). ${ }^{3} \mathrm{~A}$ terrace is etched into a SI InP before epitaxy, with $\mathrm{Si}_{3} \mathrm{~N}_{4}$ as etching mask. The typical height of the terrace is $45 \mu \mathrm{m}$. With the selective LPE, growth takes place only at the side wall and on the bottom of the terrace, and not on the top of the terrace since it is masked with $\mathrm{Si}_{3} \mathrm{~N}_{4}$. Three LPE layers, two InP cladding layers, and one quaternary InGaAsP active layer are grown as shown in Fig. 1. Note that the lateral growth of all three layers terminates at the wall of the terrace, in contrast to the continuous growth of all layers in the terrace

\footnotetext{
a) Permanent address: Chengdu Institute of Radio Engineering, People's Republic of China.
}

structure on conductive substrates. ${ }^{4,5}$ The laser reported here is also different from the groove lasers reported earlier, ${ }^{3}$ because the quaternary layer does not terminate abruptly at both sides of the active region, but is continuous on one side and tapers gradually (see Fig. 1). This provides a weaker optical waveguide which is desirable for increased mode stability. Also, scattering of light by irregularities in the sidewalls of the waveguide is minimized which results in smooth far field patterns for this structure. $P$ contacting is achieved by $\mathrm{Zn}$ diffusion from the side as shown in Fig. 1 . The current flows laterally from the $p$ contact to the junction where injection of carriers takes place. The forward bias leakage of carriers is minimized, as part of the wall of the terrace is still semi-insulating. As a result, good current confinement is achieved in the present structure.

Due to the fact that the LPE layers tend to grow faster near the concave surface than on the flat surface, together with the fact that the growth rate is higher on a surface which is adjacent to a masked area, the first InP layer prefers to grow near the corner of the terrace. Therefore the subsequent InGaAsP (active) layer always grows in a curved shape near the wall. A SEM photograph of the structure is shown in Fig. 2. The variations in active layer thickness result in a lateral variation of the effective index of refraction. ${ }^{6.7}$ In our case, as the active layer and thus the effective index of refraction taper sideways, a real index waveguide is resulted near the wall of the terrace. By optimizing the waveguide parameters such as thickness of the first InP layer, thickness of the active layer, and degree of tapering, lasers with excellent performances are obtained, as described below.

Based on the foregoing waveguiding structure, stabilized single mode lasers can be fabricated in two ways. The first version $(T-1)$ of terrace laser is a transverse-junction-

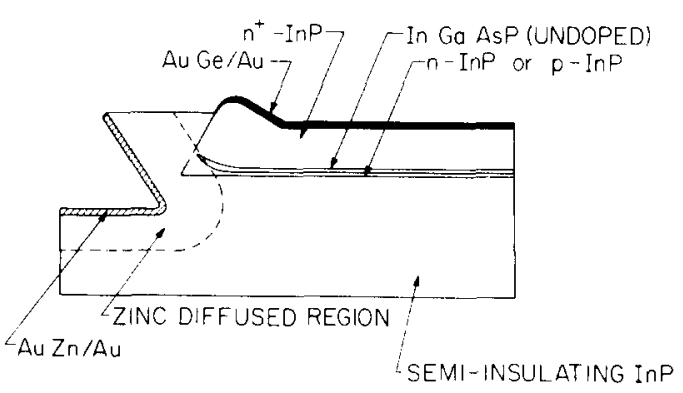

FIG. 1. Schematic cross section of the terrace laser on semi-insulating InP. 


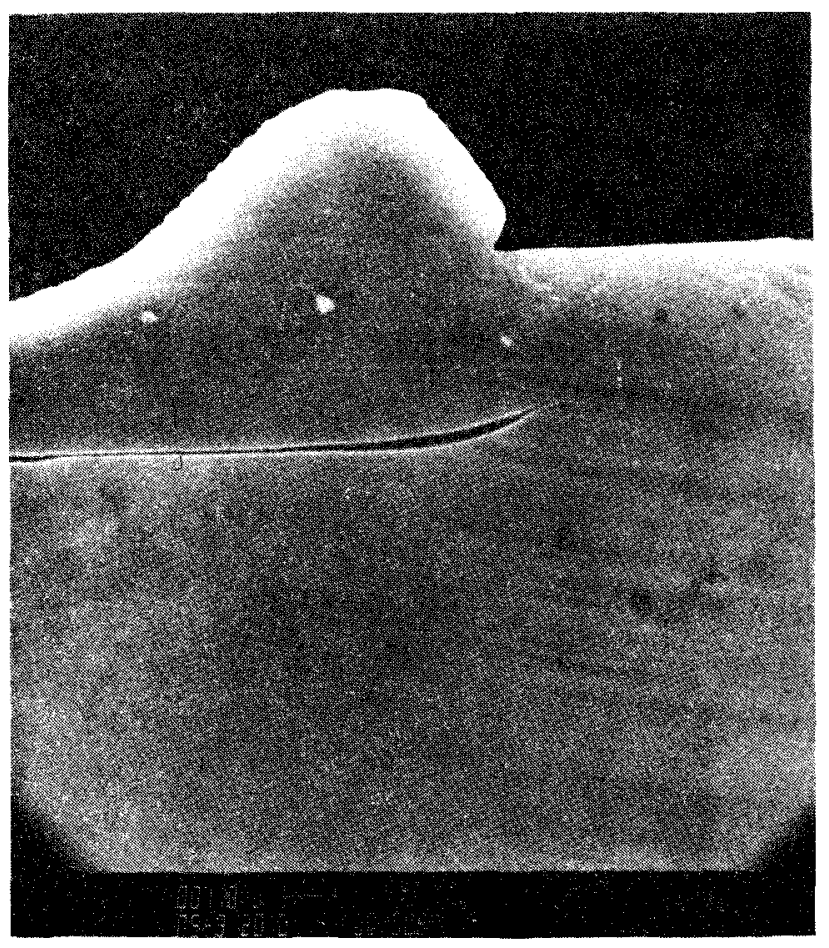

$2 \mu \mathrm{m}$

FIG. 2. A SEM photograph of the terrace laser on semi-insulating InP.

stripe type laser. ${ }^{8}$ The layers are grown as shown in Fig. 1 with the first layer $n$ type. The typical doping concentration and thickness of the LPE layers are: $n$-InP confinement layer (undoped, background electron concentration is $4-9 \times 10^{16}$ $\mathrm{cm}^{-3} ; 0.5 \mu \mathrm{m}$ thick), InGaAsP active layer (undoped, $0.2-$ $0.3 \mu \mathrm{m}$ thick at the thickest part), $n^{+}$-InP confinement layer (Sn-doped, $2 \times 10^{18} \mathrm{~cm}^{-3} ; 2-3 \mu \mathrm{m}$ thick). After LPE, a $\mathrm{Zn}$ diffusion is performed laterally with the diffusion front penetrating into the active region to form a transverse homojunction. The carrier injection in this case occurs predominantly across the lower band gap InGaAsP homojunction. By optimizing the thickness of the active layer, and the location of the transverse junction inside the active layer, low threshold current and stable mode behavior can be achieved. If the transverse junction is located near the center of the effective waveguide, the distribution of the injected carrier concentration, and thus the optical gain profile, will also peak there. This will favor the fundamental mode operation due to the maximum overlap of this mode with the gain profile. As the optical power increases, the gain profile becomes progressively narrower due to the decrease of the stimulated lifetime and thus of the effective carrier diffusion length. This effect provides stabilization for the fundamental mode. Some theoretical analysis of the injection mode will be presented elsewhere.

In the second version (T-2), the geometry is the same as the $T$ - 1 laser except the first InP layer is $p$-type ( $\mathrm{Zn}$-doped, 2$\left.5 \times 10^{17} \mathrm{~cm}^{-3}\right)$. In addition, the growth time of the first $p$ InP layer is reduced such that the first layer only grows near the corner of the terrace. The subsequent lateral $\mathrm{Zn}$ diffusion penetrates the $p$-InP layer and not the quaternary layer. The

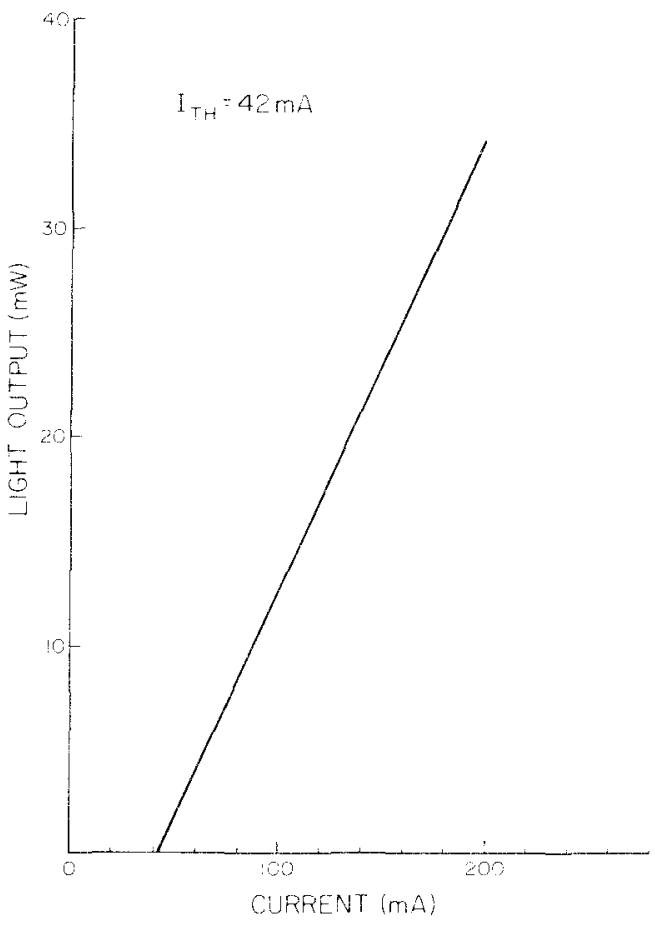

FIG. 3. $L-I$ characteristics of a T-2 laser, $I_{\mathrm{TH}}$ is $42 \mathrm{~mA}$.

above conditions result in the reduction of the effective width of the active waveguide, as the injection of holes takes place mainly from the $p$-InP near the corner to the active layer. As a result, relatively low threshold current is obtained.

Typical T-1 lasers employ a 5- $\mu \mathrm{m}$ high terrace, with a maximum active region thickness of $0.2-0.3 \mu \mathrm{m}$. The transverse junction is placed $1-1.5 \mu \mathrm{m}$ away from the edge of the active layer. This results in a stable single transverse mode operation. Lasers with cavity lengths of $250 \mu \mathrm{m}$ possess threshold current in 50-80 $\mathrm{mA}$ range. The lasing wavelength is $1.3 \mu \mathrm{m}$. The light output increases linearly with the injection current up to four times threshold current. External differential quantum efficiency is about $38 \%$ for both facets. Stable single mode operation at over 10 times threshold current and a power output of $156 \mathrm{~mW} /$ facet have been ob-

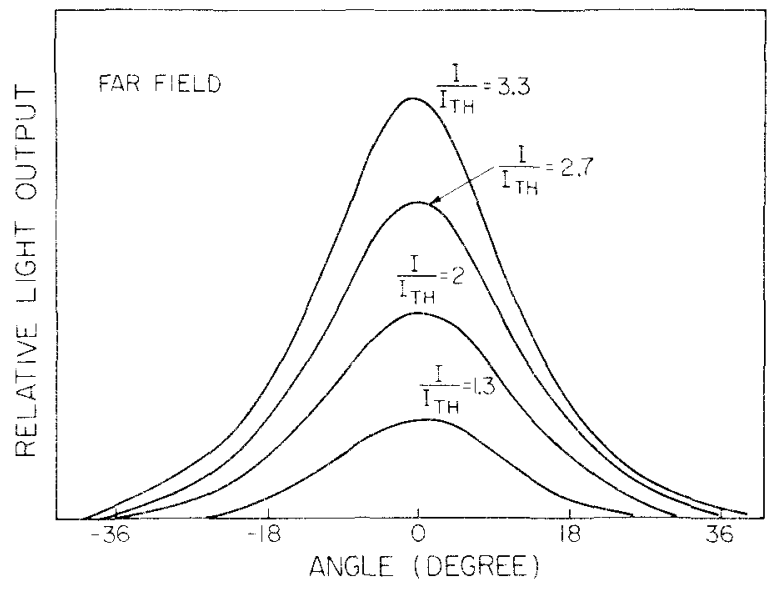

FIG. 4. A far field pattern at pulsed operation of a $\mathrm{T}-2$ laser. 
served with 50 -ns pulses. T-2 lasers, with cavity lengths of $250 \mu \mathrm{m}$, have threshold current of $35-50 \mathrm{~mA}$. A typical light versus current characteristics of the laser is depicted in Fig. 3. The characteristic is kink free, and is linear up to four times threshold current. The external differential quantum efficiency is about $45 \%$ for both facets. The lasers operate in a single fundamental mode up to high injection current level (e.g., $I>5 I_{\text {TH }}$ ), and a power output of $170 \mathrm{~mW} /$ facet has been observed at pulsed operation. A typical far field pattern of the laser is shown in Fig. 4.

In conclusion, terrace In GaAsP lasers on semi-insulating InP have been demonstrated. The fabrication processes involve only a single step LPE. Two versions of these lasers are fabricated. The lasers have relatively low threshold current, and show stable single mode operation with appreciable high power capability and well-behaved modal properties.
This work is supported by the Office of Naval Research and the National Science Foundation (Optical Communication Program).

${ }^{1}$ D. Botez, J. Opt. Commun. 1, $42(1980)$

${ }^{2}$ S. Wang, C. Y. Chen, A. S. Liao, and L. Figueroa, IEEE J. Quantum Electron. QE-17, 453 (1981).

${ }^{3}$ K. L. Yu, U. Koren, T. R. Chen, and A. Yariv, IEEE J. Quantum Electron QE-18, 817 (1982).

${ }^{4}$ T. Sugino, K. Ito, M. Wada, H. Shimizu, and I. Teramoto, IEEE J. Quantum Electron. QE-15, 714 (1979).

${ }^{5}$ K. Moriki, K. Wakao, M. Kitamura, K. Iga, and Y. Suematsu, Jpn. J. Appl. Phys. 19, 2191 (1980).

${ }^{6} \mathrm{H}$. Kogelink, IEEE Trans. Microwave Theory Tech. MTT-23, 7 (1975). ${ }^{7}$ W. Streifer and E. Kapon, Appl. Opt. 18, 3724 (1979).

${ }^{8}$ W. Susaki, T. Tanaka, H. Kan, and M. Ishii, IEEE J. Quantum Electron. QE-13, 587 (1977). 